\title{
Optimal Needle Placement for Ultrasound-Guided Knee Joint Injections or Aspirations
}

\author{
Boqing Chen ${ }^{1}$, Lawrence P Lai ${ }^{1 *}$, Nitin Putcha ${ }^{2}$, Todd P Stitik ${ }^{1}$, Patrick M Foye ${ }^{1}$ and Joel A DeLisa \\ ${ }^{1}$ New Jersey Medical School, Department of Physical Medicine and Rehabilitation, Newark, NJ, USA \\ ${ }^{2}$ University of Miami Miller School of Medicine, Miami, Florida, USA
}

\begin{abstract}
Although the inferomedial and inferior midline approaches are commonly used for intra-articular injection or aspiration of the knee joint, they may not be ideal for optimal needle placement. With these approaches, the fat pad may be contacted, which may lead to the injection of medication in the incorrect region, cause severe pain during the injection procedure, or present a false sign of low yield of fluid aspiration. Therefore, the objective of this study was to compare various approaches for knee injection and aspiration to identify the optimal one for needle placement. In this retrospective study, the ultrasound images of bilateral knee joints in 33 consecutive patients with knee pain were studied. This patient population was divided into two groups: Group A with knee effusions $(n=10)$ and Group B without knee effusions $(n=23)$. The results showed that among $90 \%$ of patients in Group A and $100 \%$ of patients in Group B, the superolateral view allowed visualization of the greatest amount of intra-articular knee fluid and the least amount of fat pad. In conclusion, the ultrasound-guided superolateral approach appears to be an optimal approach for knee intra-articular injection or aspiration compared to the conventional inferomedial and inferior midline approaches.
\end{abstract}

Keywords: Knee; Ultrasound; Intra-articular injection; Arthrocentesis

\section{Introduction}

The knee joint is one of the most commonly affected locations for various musculoskeletal diseases, such as knee osteoarthritis [1], seronegative spondy loarthropathies, infectious etiologies and trauma. These conditions could not only impair functionality of the knee joints but also cause pain in these areas. To facilitate diagnosis of the underlying disease, arthrocenteses are sometimes needed, during which sufficient joint fluid is obtained to ensure accurate laboratory assessments. At the same time, intra-articular knee injections with corticosteroids are often performed to relieve pain among patients who failed systemic non-steroid anti-inflammatory drugs (NSAIDs). Therefore, knee injection and aspiration procedures often play an important role in diagnosis and therapeutic pain relief for the knee joints.

Both procedures (aspiration and injection) require accurate placement of the needle in the intra-articular space. Therefore, understanding the anatomy of the knee is critical in the success of these procedures. The knee joint consists of three articulations two femorotibial articulations (lateral and medial), and one patello-femoral articulation between the patella and the femur $[2,3]$. The stability of knee joint depends on the strength of the associated musculature, ligamentous structures and tendons. The knee also contains three fat pads which provide extra support for the joint. superior The infra-patellar fat pad, also known as Hoffa's fat pad, lies beneath the patella and occupies the majority of the inferior joint line of the knee $[2,3]$. The pre-femoral fat pad is located along the femur and deep to the suprapatellar bursa. Lastly, the quadriceps fat pad is located to the suprapatellar bursa but inferior to the rectus femoris and vastus intermedius. Accurate placement of the needle is critical to maximize the amount of diagnostic fluid obtained, optimize the effectiveness of injected medications, and improve patient comfort. Therefore, it is important to avoid tendons and fat pads during the needle placement.

Common approaches of needle placements during arthrocenteses and intra-articular knee injections include inferomedial and inferior midline approaches. When utilizing these approaches, some structures would be contacted before the needle reaches the intra-articular space, e.g., medial meniscus and Hoffa's fat pad [4]. The latter is more consequential because it may significantly reduce the amount of the joint fluid that can be obtained during arthrocenteses. Furthermore, in therapeutic joint injections, the needle tips may be embedded in the fat pad or the intra-articular swollen synovium. Therefore, the medication injected may not reach true joint space. Visualization of intra-articular fluid significantly improves the accuracy of the needle placement into the joint space without contacting the surrounding structures. Therefore, as part of our routine patient care, we utilize ultrasound imaging to view the knee joint from various angles in order to determine which approach is most optimal, i.e., allowing a view of the greatest amount of intra-articular fluid and the least amount of surrounding fat. We reported our findings and recommended the optimal approach for knee joint injection and aspiration.

\section{Methods}

Approval from the sponsoring institutional review board was obtained prior to the study. Patients who presented to our clinic with knee pain from July 27, 2009 to August 23, 2009 and who had ultrasound images of bilateral knee joints were included for this study. The underlying diseases for knee pain were heterogeneous, including osteoarthritis, meniscal tears, bursitis and trauma. Eligible patients were divided into two groups: Group A with knee effusions and Group B without knee effusions. Ultrasound images for all affected knees were taken from 6 different views: superolateral, superomedial, superior

*Corresponding author: Lawrence P Lai, Department of Physical Medicine and Rehabilitation, New Jersey Medical School, NJ, USA, Tel: 1 973-972-4783; E-mail: lawrencelai1998@yahoo.com

Received September 15, 2014; Accepted October 24, 2014; Published October 26,2014

Citation: Chen B, Lai LP, Putcha N, Stitik TP, Foye PM, et al. (2014) Optimal Needle Placement for Ultrasound-Guided Knee Joint Injections or Aspirations. J Trauma Treat 3: 216. doi:10.4172/2167-1222.1000216

Copyright: ( $) 2014$ Chen B, et al. This is an open-access article distributed unde the terms of the Creative Commons Attribution License, which permits unrestricted use, distribution, and reproduction in any medium, provided the original author and source are credited. 
Citation: Chen B, Lai LP, Putcha N, Stitik TP, Foye PM, et al. (2014) Optimal Needle Placement for Ultrasound-Guided Knee Joint Injections or Aspirations. J Trauma Treat 3: 216. doi:10.4172/2167-1222.1000216

midline, inferolateral, inferomedial and inferior midline views. A representative sample of each view was shown within Figures 1-6. All the images were retrospectively reviewed with the focus on the visualization of the relative amount of synovial fluid compared to the amount of fat pad. The transducer used was from the Merlin 1101 (BK Medical() with frequency from 6 to $12 \mathrm{MHz}$.

In order to determine the best approach for the knee injection or aspiration, patients were placed in the supine position with the knee in approximately 30 degrees of flexion using a bolster underneath the popliteal fossa. The aforementioned six views of ultrasound imaging were taken for the affected knees. The view which showed the best access to the joint space was then chosen for the injection approach. The various images were retrospectively analyzed with respect to the visualized amount of intra-articular fluid and the amount of fat pad.

\section{Results}

A total of 33 patients met the eligibility criteria and thus were included in the current study. Group A (effusion) had 10 patients and Group B (no effusion) had 23 patients. In group A, the superolateral approach showed the largest amount of intra-articular fluid with the least amount of fat pad in $90 \%$ of patients. Approximately $10 \%$

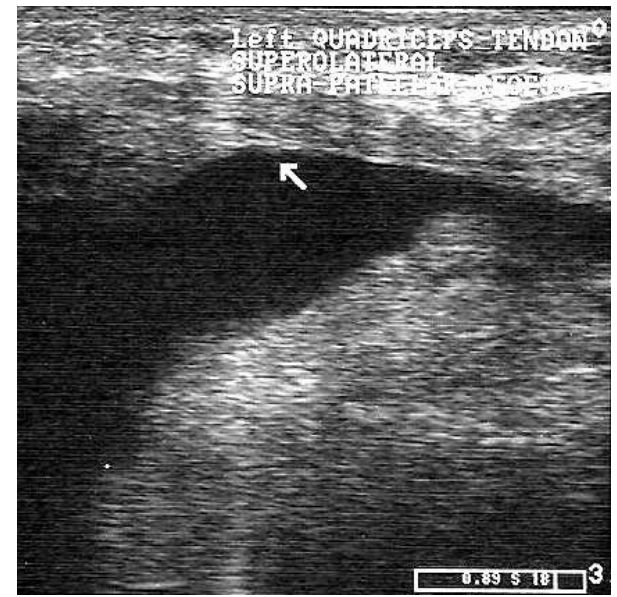

Figure 1: Ultrasound (US) scan revealed the most amount of intra-articular fluid with the least amount of fat pad with the superolateral approach.

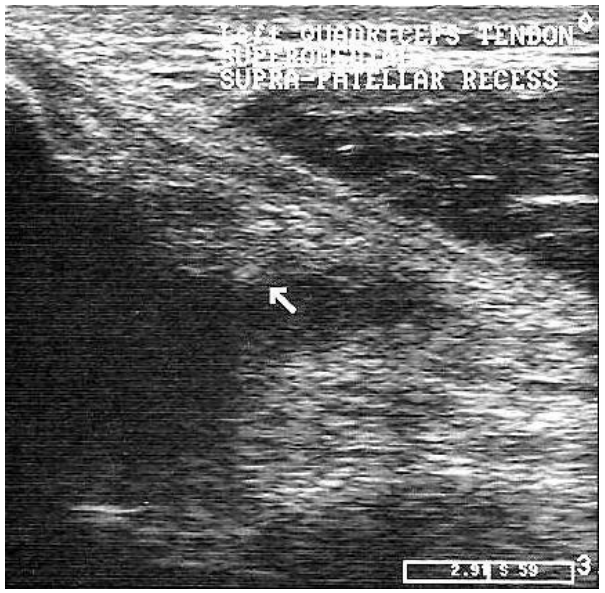

Figure 2: Ultrasound (US) scan view with the superomedial approach.

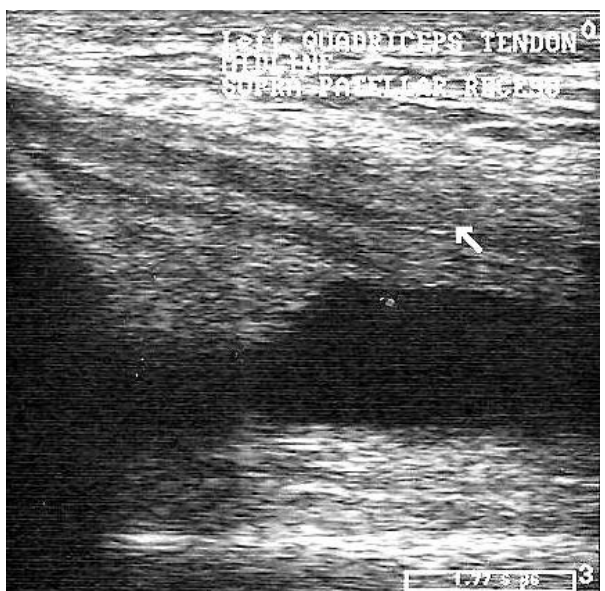

Figure 3: Ultrasound (US) scan view with the superior middle approach.

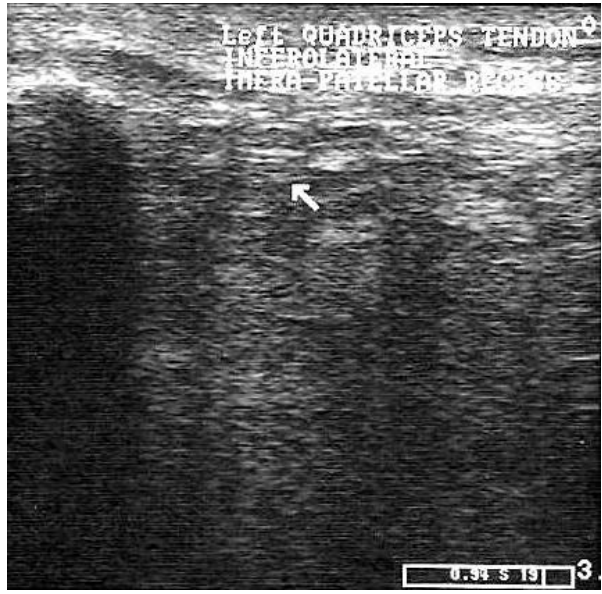

Figure 4: Ultrasound (US) scan view with the inferolateral approach.

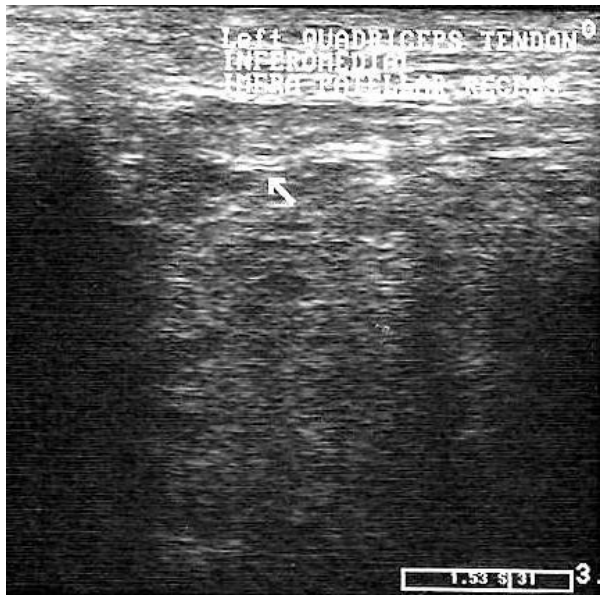

Figure 5: Ultrasound (US) scan view with the inferomedial approach.

of individuals in this group yielded a small amount of fluid that was observed in the inferior midline approach (Table 1). The amount of fluid observed in the inferior midline approach seems to be irrelevant of 


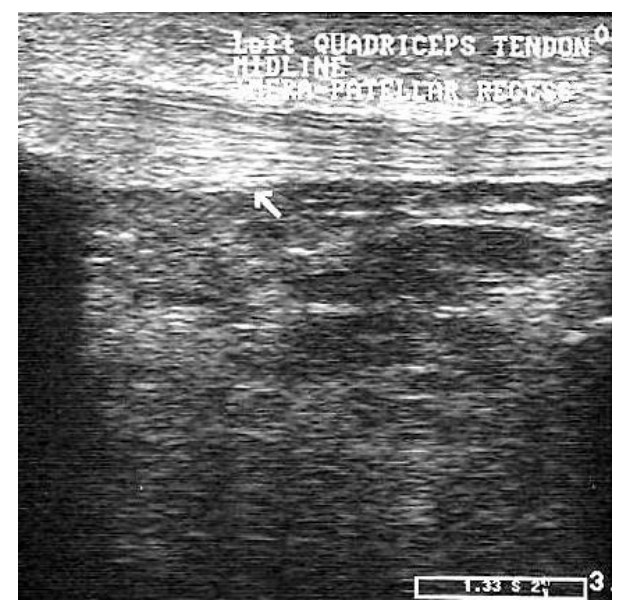

Figure 6: Ultrasound (US) scan view with the inferior midline approach.

the patients' diagnoses. It is important to note, however, that Hoffa's fat pad consistently filled the infrapatellar space shown in the inferolateral, inferomedial and inferior midline approaches even when a large amount of knee effusions were observed in the suprapatellar recess in the superolateral, superomedial and superior midline approaches.

Consistent with the findings in group A, the superolateral approach also showed the largest amount of intra-articular fluid with the least amount of fat pad in $100 \%$ of the patients in group B. Given the consistent findings of fat pad visualization when the knee is imaged using an inferior approach (i.e., inferolateral, inferomedial or inferior middle), a needle placed using the inferior approach frequently may encounter fat pad even in the presence of a large amount of effusion. This may result in false negative aspiration of knee joint fluid or deposition of the medication into Hoffa's fat pad rather than in the intra-articular space.

In summary, both groups (with and without effusion) showed that the superolateral view yielded the greatest amount of intra-articular fluid and the least amount of surrounding fat pad tissue (quadriceps and infra-patellar fat pads). Additionally, the joint space can still be visualized beneath the superolateral aspect of the patella. Hence, the superolateral approach with the needle entering just parallel to the undersurface of the patella may be the optimal approach for intraarticular injection or aspiration.

The actual needle joint entry using the superolateral approach was subsequently confirmed in a patient for whom fluoroscopic guidance was used instead of ultrasound due to the unavailability of ultrasound on that case. In this image, the contrast injection under the patella and the contrast diffusion into the joint space could be clearly observed (Figure 7).

\section{Discussion}

Arthrocenteses and intra-articular knee injections are common procedures used in the diagnosis of underlying diseases affecting the knee and relief of the pain in the joint. The conventional approaches include inferomedial and inferior midline. However, previous studies have shown that these two approaches may not be efficient because there is a high chance for the needle to contact the surrounding tissues before reaching the intra-articular space [5].

The accuracy of knee joint injections with various approaches has been studied previously by tracing the fluoroscopic contrast in the intra-articular space [5]. This study demonstrated 93\% accuracy of intra-articular needle placement using the lateral midpatellar approach [5]. In another study on knee injection using human cadavers, the anterolateral approach had the highest accuracy (85\%) of intraarticular needle placement as compared with the anteromedial, medial midpatellar and lateral midpatellar approaches [6]. Although a previous study suggested that ultrasound guidance could improve the accuracy of joint injections [7], to date, there have been no published studies using ultrasound guidance to assess various approaches for knee joint injection and aspiration and to identify the optimal approach.

In this study, we reviewed the ultrasound images of bilateral knee joints among patients with knee pain. Among the six views under the ultrasound, the superolateral view showed the greatest amount of joint fluid and the least amount of fat pad in most of the patients. Therefore, the superolateral approach is recommended as the optimal approach for knee injection and aspiration. This approach should expose the needle tip to the greatest amount of synovial fluid but the least amount of fat pad. With a superolateral approach, the patient should be placed in the supine position with the knee in 30 degrees of flexion using a bolster underneath the popliteal fossa. Because of the consistent presence of fluid in the superomedial and the superior middle areas in the patients with knee joint effusion, the superomedial approaches and superior midline approach through quadriceps tendon can also be used for intra-articular injection or arthrocentesis. However, the possibilities for the needle trauma in the vastus medialis muscle (a medial knee joint stabilizer) with the medial approach and the needle trauma in the quadriceps tendon through the midline approach makes these two approaches less desirable than the superolateral approach. Additionally, ultrasound guidance should be used, when available, in order to increase the accuracy of intra-articular needle placement. The inferomedial and inferior midline approaches for intra-articular needle placement may be less reliable and potentially more painful because the diffusely located Hoffa's fat pad could block the needle.

A limitation of the study is that we did not compare the accuracy of the needle entry for each approach. However, being able to visualize the fat pad with ultrasound guidance would make these comparisons unnecessary and unethical. Significant injuries can occur from unsuccessful needle entries in the attempt to obtain the knee joint aspirate. Another limitation is the limited sample size. However, the statistical analysis revealed a significant outcome. Finally, positioning of the patient either sitting or supine was not compared. However, a study by Zhang et al. [8] revealed that in the supine position, more joint fluid was aspirated from the knee joint [8].

\section{Conclusions}

Based on the ultrasound-guided views, the superolateral view is the

\begin{tabular}{|c|c|c|c|c|c|c|}
\hline & Superior midline & Superolateral & Superomedial & Inferior midline & Inferolateral & Inferomedial \\
\hline & $\begin{array}{l}\text { Joint Fluid? } \\
\text { Yes No }\end{array}$ & $\begin{array}{l}\text { Joint } \text { Fluid? } \\
\text { Yes No }\end{array}$ & $\begin{array}{l}\text { Joint Fluid? } \\
\text { Yes No }\end{array}$ & $\begin{array}{l}\text { Joint Fluid? } \\
\text { Yes No }\end{array}$ & $\begin{array}{l}\text { Joint } \text { Fluid? } \\
\text { Yes No }\end{array}$ & $\begin{array}{l}\text { Joint Fluid? } \\
\text { Yes No }\end{array}$ \\
\hline $\begin{array}{l}\text { Group A } \\
\text { Group B }\end{array}$ & $\begin{array}{l}10 \\
23\end{array}$ & $\begin{array}{l}10 \\
23\end{array}$ & $\begin{array}{l}10 \\
23\end{array}$ & $\begin{array}{l}1 \\
0\end{array}$ & $\begin{array}{ll}0 & 10 \\
0 & 23\end{array}$ & $\begin{array}{l}0 \\
0\end{array}$ \\
\hline
\end{tabular}

Table 1: Number of patients with intra-articular knee fluid in 6 ultrasound-guided views between two groups: Group $A($ effusion, $N=10$ ) and Group $B$ (no effusion, $N=23$ ) 
Citation: Chen B, Lai LP, Putcha N, Stitik TP, Foye PM, et al. (2014) Optimal Needle Placement for Ultrasound-Guided Knee Joint Injections or Aspirations. J Trauma Treat 3: 216. doi:10.4172/2167-1222.1000216

Page 4 of 4

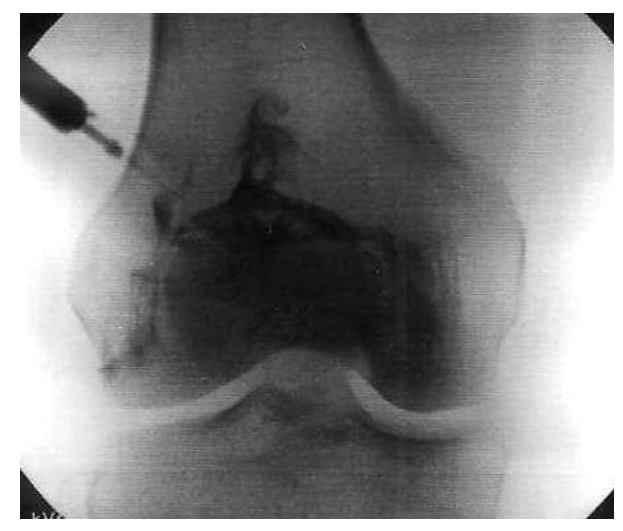

Figure 7: Fluoroscopic-guided knee joint injection. Needle placement is visualized with the superolateral approach.

most ideal position for knee joint injections or aspirations compared to the superior midline, superomedial, inferior midline, inferolateral and inferomedial views with the knee in $30^{\circ}$ of flexion using a bolster underneath the popliteal fossa. This study shows that the ultrasoundguided superolateral approach allows for the best view of the intraarticular fluid with the least amount of fat pad, and thus optimizes the needle placement. Furthermore, when available, ultrasound guidance is recommended for routine knee joint injections and aspirations so that soft tissues, such as the fat pads that may not be palpated by physical examination could be visualized in the imaging.

\section{References}

1. Oliveria SA, Felson DT, Reed JI, Cirillo PA, Walker AM (1995) Incidence of symptomatic hand, hip, and knee osteoarthritis among patients in a health maintenance organization. Arthritis Rheum 38: 1134-1141.

2. Moore KL, Dalley AF, Agur AMR (2010) Clinically Oriented Anatomy (6thedn), Lippincott Williams and Wilkins, London.

3. Ahuja AT, Antonio GE, Griffith JF, Ho SSM, Wong KT, et al. (2007) Diagnostic and Surgical Imaging Anatomy, 58-75.

4. LaPrade RF (1998) The anatomy of the deep infrapatellar bursa of the knee. Am J Sports Med 26: 129-132.

5. Jackson DW, Evans NA, Thomas BM (2002) Accuracy of needle placement into the intra-articular space of the knee. J Bone Joint Surg Am 84-84A: 1522-7.

6. Cunnington J, Marshall N, Hide G, Bracewell C, Isaacs J, et al. (2000) A randomized, double-blind, controlled study of ultrasound-guided corticosteroid injection into the joint of patients with inflammatory arthritis. Arthritis Rheum. 62: 1862.

7. Esenyel C, Demirhan M, Esenyel M, Sonmez M, Kahraman S, et al. (2007) Comparison of four different intra-articular injection sites in the knee: a cadaver study. Knee Surg Sports Traumatol Arthrosc 15: 573-577.

8. Zhang Q, Zhang T, Lv H, Xie L, Wu W, et al. (2012) Comparison of two positions of knee arthrocentesis: how to obtain complete drainage. Am J Phys Med Rehabil 91: 611-615. 